\title{
Die Gründung des Zürcher Lehrstuhles für Anthropologie
}

Von Hans-Konrad Schmutz

I

Die Schaffung neuer Institutionen gehört im organisatorischen Bereich zu den wichtigsten Merkmalen naturwissenschaftlicher Anthropologie des 19. Jahrhunderts. Die zunehmende Professionalisierung, gefördert durch die Einbeziehung des Faches in die bestehenden Promotionsordnungen ist eng damit verknüpft.

Die Gründung eigener, d.h. nur der anthropologisch-ethnographischen Forschung gewidmeten Fachgesellschaften stellt eine wichtige Etappe dieses Weges dar. Die 1859 gegründete Société d'Anthropologie de Paris sei als Beispiel genannt. Sie stellt eine für die Weiterentwicklung und Standardisierung quantitativer Methoden wichtige Gruppierung dar. Hatte sich diese Pariser Gesellschaft aus der Société de Biologie heraus entwickelt, so fußte die zehn Jahre später gegründete Deutsche Gesellschaft für Anthropologie, Ethnologie und Urgeschichte auf der Versammlung der Deutschen Naturforscher und $\ddot{\text { Arzte }}{ }^{1}$. Rudolf Virchow, den man zurecht als Organisator der anthropologischen Forschung in Deutschland bezeichnet hat ${ }^{2}$, war nicht nur federführend in der nationalen Organisation, sondern auch langjähriger Präsident der Berliner Gesellschaft für Anthropologie, Ethnologie und Urgeschichte, die man zu den bedeutendsten anthropologischen Lokalvereinigungen Deutschlands vor dem Ersten Weltkrieg zählt. Schon 1870 bestand der Berliner Verein aus 138 ordentlichen Mitgliedern ${ }^{3}$. Er verfügte auch bald über eine eigene Sammlung und eine eigene Bibliothek.

Aus den vereinseigenen Sammlungen entstanden an mehreren Orten Museen. So konnte beispielsweise die vom Schleswig-holsteinischen anthropologischen Verein gegründete Sammlung 1888 als Museum von der Universität Kiel übernommen werden. Andernorts, wie etwa in Hamburg, bildete die ethnographische Sammlung der Stadtbibliothek den Grundstock. Nicht zu vergessen ist auch die Bedeutung von Privatsammlungen, wie die der reichen Japansammlung Siebold in München ${ }^{4}$.

Viele dieser Museen entstanden in den siebziger und achtziger Jahren, nicht zuletzt im Zusammenhang mit den aufkeimenden Kolonialbestrebun- 
gen des neugegründeten Reiches. Bald folgte der Ruf nach geeigneten, für den Dienst in den überseeischen Gebieten vorbereiteten, d.h. mit Ethnographie und Rassenkunde vertrauten Beamten und Offizieren. Die an der Kolonialpolitik interessierten Kreise unterstützten deshalb die Forderung, anthropologische Fächer in den Lehrplan der Hochschulen aufzunehmen. Die entsprechenden Vorlesungen von Felix von Luschan (1854-1924) und Adolf Bastian (1826-1905) an der Berliner Universität wurden regelmäßig im Deutschen Kolonialblatt angezeigt ${ }^{5}$. Weiter faßte die Deutsche Kolonialgesellschaft an ihrer Straßburger Tagung im Dezember 1899 den Entschluß, bei den Regierungen die vermehrte Schaffung von Lehrstühlen zu fordern ${ }^{6}$.

Wissenschafter haben ähnliche Argumente vorgebracht. Wilhelm von Waldeyer (1836-1921) hat beispielsweise 1899 mit besonderem Nachdruck auf den politisch-wirtschaftlichen Nutzen dieser Wissenschaftszweige hingewiesen:

«Ich will vielmehr darauf hinweisen, daß in Folge der ungemein erweiterten Handelsbeziehungen aller Völker, die Pflege der Anthropologie für unseren Erdtheil, der wenigstens in seinen westlichen Gliedern, und darunter auch in Deutschland, das nicht mehr in hinreichender Menge zu erzeugen vermag, was des Leibes Nahrung und somit die ganze Existenz ermöglicht, ungemein wichtig, ja nothwendig wird. Und was soll ich erst von denjenigen Staaten sagen, welche Colonialbesitz erworben haben und zu erwerben trachten. Niemand sollte dort, wenigstens in administrative Stellung hinausgehen, der nicht hinreichend ethnologisch geschult wäre. Wer eines will, darf auch das andere nicht lassen! Vor allem müssen wir in Deutschland, wenn wir den Wettbewerb mit den großen anderenHandels- und Colonialmächten aushalten wollen, allen Ernstes darauf bedacht sein, für einen besseren Unterricht in ethnologisch-anthropologischer Beziehung zu sorgen und darüber zu wachen, daß junge Forscher herangebildet werden, die, wenn die Jetztlebenden müde geworden sind, das Zeug dazu haben, in die Lücken zu treten und, besser hoffentlich noch als wir, das fortführen, was wir begonnen haben. Das kann, meines Erachtens, aber nur erreicht werden durch die Einfügung der anthropologischen Disciplinen als integrierte Bestandteile in den Universitätsunterricht!’ ${ }^{7}$

Als Waldeyer mit dieser Rede die gemeinschaftlichen Sitzungen der Deutschen und der Wiener anthropologischen Gesellschaft eröffnet hatte, bestand innerhalb Deutschlands lediglich in München eine ordentliche Professur für Anthropologie. In der bayrischen Hauptstadt war 1884 aus dem alten Lehrauftrag für allgemeine Naturgeschichte die neue Professur gebildet worden ${ }^{8}$. Selbst an der Berliner Universität, an der Bastian sein erstes ethnographisch-anthropologisches Kolleg schon 1868 gehalten hatte, wurde erst 1900 eine außerordentliche Professur für Völkerkunde mit besonderer Berücksichtigung der Anthropologie geschaffen ${ }^{9}$. 
Die skizzierten Entwicklungsschritte können auch bei der Etablierung der anthropologischen Wissenschaften an der Universität Zürich nachgewiesen werden.

Außerhalb der Universität kam es 1888 zur Gründung der ethnographischen Gesellschaft. Nicht nur Wissenschafter wie Albert Heim (1849-1937) und Otto Stoll (1849-1922), sondern auch Vertreter des Handels gehörten zu den Initianten. Die junge Gesellschaft konnte bereits ein Jahr nach ihrer Gründung ein kleines Museum eröffnen. Die kaufmännische Gesellschaft hatte hierzu den nötigen Ausstellungsraum in der Kuppel der Börse zur Verfügung gestellt.

Parallel dazu begann man den anthropologischen Fächern auch an der Universität vermehrt Aufmerksamkeit zu schenken. Für den Zeitraum zwischen 1860 und der Gründung der ethnographischen Gesellschaft lassen sich vereinzelte Ankündigungen anthropologischer Vorlesungen nachweisen. Die Vorlesung «Abstammung, Schädelbildung und Raçentypen des Menschen», welche der Privatdozent und spätere Reiseschriftsteller Johann Theodor Gsell Fels (1818-1898) im Sommersemester 1868 halten wollte, mag als Beispiel dienen. Er scheint allerdings keine Hörer gefunden zu haben ${ }^{10}$. Als er ein Semester später über die Naturgeschichte des Menschen sprechen wollte, blieben die Studenten ebenfalls weg. Der Geograph Jakob Egli (1825-1896) verzeichnete mit seiner Vorlesung über physische Geographie, die auch eine kurzgefaßte Rassenkunde beinhaltete, einen besseren Erfolg. Besonders interessant ist die Tatsache, daß seine Vorlesung über die deutschen Kolonien im Sommersemester $1888 \mathrm{zu}$ den hervorragend besuchten Veranstaltungen der Fakultät gehörte ${ }^{11}$. Dies mag das allgemeine Interesse an Kolonialfragen illustrieren. Ein gutes Echo fand auch Albert Heims Vorlesung über die Urgeschichte des Menschen, die er in modifizierter Form während zwanzig Jahren las. Sie enthielt allerdings nur spärliche Angaben zu Paläoanthropologie und Rassenkunde ${ }^{12}$. Den Schwerpunkt bildete vielmehr die Darstellung materieller Hinterlassenschaften, wie etwa die Herstellung und Form nachsteinzeitlicher Werkzeuge. Wenig erstaunen mag auch das Interesse des Dozenten an einer ausgedehnten Schilderung der schweizerischen Pfahlbausiedlungen. Diese Gewichtung Heims widerspiegelt sich auch in einer erhaltenen Studentennachschrift aus dem Wintersemester $1875 / 76^{13}$. 
Das Gründungsmitglied der ethnographischen Gesellschaft Otto Stoll hat sich 1884 an der ersten Sektion der Philosophischen Fakultät mit einer Studie zur Ethnographie der Republik Guatemala habilitiert. Er kündigte noch für das Sommersemester 1884 eine Vorlesung mit dem Titel «Anthropologie und Ethnographie» an. Er las über dieses Thema bis 1891 mit gutem Erfolg. Dabei konnte er meist eine überdurchschnittliche Beteiligung verzeichnen. Soweit sich der Aufbau der Vorlesung anhand des Stollschen Nachlasses rekonstruieren läßt, bestand sie gut zur Hälfte aus einer biologisch verstandenen, deutlich von Völkerkunde und Urgeschichte geschiedenen Anthropologie ${ }^{14}$. Er vermittelte einen Einblick in aktuelle Fragen der fachinternen Auseinandersetzung indem er beispielsweise die rassenkundlichen Systeme von Quatrefages, Topinard und Kollmann einander gegenüberstellte. Demgegenüber blieb er in der Beurteilung des Neanderthalfundes zurückhaltend. Er kommentierte auch die Haeckelschen Thesen sehr vorsichtig. Stoll hat mit dieser gut besuchten Vorlesung wie auch mit seinem Eintreten für die Völkerkunde in Zürich den Weg für die weitere Entwicklung an der Universität gewiesen.

1891 stellte sich an der zweiten Sektion der Philosophischen Fakultät die Frage nach der Schaffung eines Lehrstuhles für Geographie. Sie sollte, nach den Überlegungen des Erziehungsrates, verknüpft werden mit der Berufung Stolls unter gleichzeitiger Berufung zum Extraordinarius. Innerhalb der Fakultät wurde diese Kandidatur d.h. die Betonung der völkerkundlichkulturhistorischen Seite der Geographie, von Alfred Kleiner (1849-1916) unterstützt. In entschiedener Weise trat auch der Haeckel-Schüler Arnold Lang für die Kandidatur Stolls ein. Albert Heim betonte demgegenüber die Fähigkeiten Jacob Frühs, den er als geeignet ansah:

«Die Geographie nach der Richtung der Geologie, physikalischen Geographie, Oceanographie usw. zu vertreten.» ${ }^{15}$

Mit der erfolgreichen Wahl Stolls war, wie bereits angedeutet, die der Anthropologie verwandte Seite der Geographie an der Fakultät verankert worden. Dies war mit der Grund, daß sich Rudolf Martin (1864-1925) bereits ein Jahr später für das Fach Anthropologie habilitieren konnte. Wiederum war es Stoll, der zusammen mit dem Zoologen Arnold Lang die eingereichte Schrift «Zur Physiologischen Anthropologie der Feuerländer» zu begutachten hatte. Wenngleich er sich an einigen hypothetischen Schlußfolgerungen Martins stieß, so stimmte er doch mit Arnold Lang darin überein, daß der 
Habilitand Ergebnisse vorzuweisen habe, wie sie in ähnlicher Gründlichkeit und Ausführlichkeit für eine außereuropäische Rasse noch kaum existiert hätten ${ }^{16}$. Er unterstrich weiter die methodische Sauberkeit des Untersuchers. Wegen seiner methodischen Beiträge zählt man heute Rudolf Martin zu den Pionieren quantitativer Verfahren in der Anthropologie. Er hatte die wesentlichen Impulse hierzu während zweier Aufenthalte an der Ecole d'Anthropologie de Paris in den Jahren 1888 und 1889 erhalten. Es gelang ihm mit seinem 1914 erstmals publizierten «Lehrbuch der Anthropologie», die quantitativen Methoden zu vereinheitlichen und einen für viele Bereiche der Anthropologie brauchbaren Katalog an Meßpunkten und Meßstrecken vorzulegen. Es wird in veränderter Fassung noch heute aufgelegt, was die Bedeutung des Werkes belegt.

Einige Stichworte zu seinem Werdegang. 1864 in Zürich-Hottingen geboren, verbrachte er seine Schulzeit im badischen Offenburg. Er begann 1884 sein Studium an der Universität Freiburg. In seinem handgeschriebenen Lebenslauf nennt er den Zoologen August Weismann als einen seiner wichtigen Lehrer ${ }^{17}$. Nach einem Studienaufenthalt in Leipzig promovierte er 1887 in Freiburg mit einer Arbeit über Kants philosophische Anschauungen in den Jahren 1762-1766. Im Juni 1890 ließ er sich, nach den bereits erwähnten Aufenthalten in Paris, endgültig in Zürich nieder, um die Habilitationsschrift auszuarbeiten. Er erhielt die Lehrbefugnis am 9. August 1892 nach Absolvierung einer Probevorlesung über Rassenvariationen am Skelett.

Im Sommersemester 1893 leitete er einen anthropometrischen Kurs und erteilte in einer einstündigen Vorlesung praktische Ratschläge zur wissenschaftlich-anthropometrischen Arbeit auf Reisen. Beide Veranstaltungen waren gut besucht. Eine eigentliche Einführung ins Studium las er im folgenden Semester. Er übernahm in diesem Wintersemester 1893/94 zusätzlich den Lehrauftrag «Grundzüge der Anatomie des Menschen für Lehramtskandidaten und andere Nichtmediziner». Später ergänzte er sein Angebot durch die Behandlung der Anthropometrie im Rahmen kriminalistischer und schulhygienischer Untersuchungen nebst entsprechender Praktikumsstunden. Diese Veranstaltungen stießen auf ein reges Interesse. Dies war wohl mit der Grund dafür, daß Otto Stoll in der Fakultätssitzung vom 8. Februar 1899 die Errichtung eines Extraordinates für Anthropologie und Anatomie für Nichtmediziner anregte. Nach Voten von Martin, Stoll und Lang einigte man sich auf einen entsprechenden Antrag, dem sich die Erziehungsdirektion mit der folgenden Argumentation anschloß: 
«Die physische Anthropologie, d.h. die Lehre von Körpermerkmalen der menschlichen Species (im zoolog. Sinne) und die vergleichende Lehre von den menschlichen Rassen habe sich im letzten Jahrzehnt immer mehr zu einer selbständigen Wissenschaft entwickelt, deren Bedeutung als Hülfsdisciplin für eine Reihe anderer Wissenschaften, vor allem der Ethnologie, der allgemeinen menschlichen Anatomie, der vergleichenden Anatomie u. der Zoologie, dann aber auch der Kriminalrechtspflege, in steter Zunahme begriffen sei.» ${ }^{18}$

Martin wurde wenig später befördert. Damit stellte sich die Frage nach der Gestaltung eines Studienganges in Anthropologie, d.h. nach einer entsprechenden Änderung der Promotionsordnung. Die Fakultät einigte sich am 17. November darauf, nebst dem Hauptfach, vergleichende Anatomie und Humananatomie zu obligatorischen Nebenfächern zu erklären. Ein drittes Nebenfach konnte der Student frei wählen. Weiter hatte der zukünftige Anthropologe Studienausweise in Geographie und Ethnologie zu erbringen.

Mit der Festlegung des Studienganges und der entsprechenden Änderung der Promotionsordnung war das Fach theoretisch an der Universität verankert. Wie gezeigt wurde, zählt damit Zürich zu den Hochschulen des deutschsprachigen Raumes, die sehr früh einen Studiengang in Anthropologie anboten. Die steigende Studentenzahl belegt, daß dieses Angebot einem Bedürfnis entsprach und bald das Fach auch praktisch verankert war ${ }^{19}$.

\section{Anmerkungen}

${ }^{1}$ Querner, Hans: Die Anthropologie auf den Versammlungen der Deutschen Naturforscher und Ärzte bis zur Gründung der Gesellschaft für Anthropologie 1869; in: Hundert Jahre Berliner Gesellschaft für Anthropologie, Ethnologie und Urgeschichte 1869-1969, p. 143-156 (Berlin 1969).

${ }^{2}$ Vgl. Ackerknecht, Erwin H.: Rudolf Virchow (Stuttgart 1957).

3 Andree, Christian: Geschichte der Berliner Gesellschaft für Anthropologie, Ethnologie und Urgeschichte 1869-1969, p.20 (Berlin 1969).

${ }^{4}$ Vgl. Bahson, Kristian: Über ethnographische Museen. Mitt. Anthropol. Ges. Wien 18 (1888) 109-164.

${ }^{5}$ Beispielsweise: Deutsches Kolonialblatt - Amtsblatt für die Schutzgebiete des Deutschen Reiches 10 (1899) 741 und 11 (1900) 293.

${ }^{6}$ Bei der Pariser Weltausstellung 1900 waren wesentlich mehr anthropologisch-ethnographische Schaustücke in den nationalen Kolonialpavillons zu sehen als in der schlecht organisierten Fachausstellung der Société d'Anthropologie de Paris. Diese Beobachtung belegt ein weit über den Fachkreis hinausgehendes Interesse.

7 Corr. Bl. Dtsch. Ges. Anthropol. Ethnol. Urgesch. 30 (1899) 74. 
${ }^{8}$ Vgl. Lexis, Wilhelm: Die Deutschen Universitäten, Bd.2, p. 120-124 (Berlin 1893).

${ }^{9}$ Schott, Lothar: Zur Geschichte der Anthropologie an der Berliner Universität. Wiss. Z. Humboldt-Univ. Berlin, Math. Nat. R. X (1961) 57-65.

${ }^{10}$ Die Information über das Zustandekommen einer Veranstaltung bzw. über die Hörerzahl ergibt sich aus dem Vergleich der Angaben im Vorlesungsverzeichnis mit denen des entsprechenden Jahresberichtes aus dem Archiv der Universität.

${ }^{11}$ Die Vorlesung «Die Deutschen Colonien» wurde von 28 Hörern belegt bei einer durchschnittlichen Studentenzahl von 10 pro Veranstaltung an der Fakultät im Sommersemester 1888.

${ }^{12}$ Handschriftenabteilung der Zentralbibliothek Zürich: Nachlaß Albert Heim II 1: Urgeschichte des Menschen.

${ }^{13}$ Handschriftenabteilung der ETH-Bibliothek Zürich: Albert Heim, Urgeschichte des Menschen, Vorlesungsnachschrift von Georg Szávits, Ing.-Schule der ETH, 3. Kurs, WS 1875/76 (bildet einen Teil von Hs. 489:31).

${ }^{14}$ Handschriftenabteilung der Zentralbibliothek Zürich: Nachlaß Otto Stoll, Ms. Z IX 156.

${ }^{15}$ Universitätsarchiv: Protokoll der Fakultätssitzung vom 20. Juli 1891.

${ }^{16}$ Staatsarchiv des Kantons Zürich: U $110 \mathrm{~s}_{2}$.

${ }^{17}$ Staatsarchiv des Kantons Zürich: U $110 \mathrm{~s}_{2}$.

${ }^{18}$ Staatsarchiv des Kantons Zürich: U 110b 2 , Mappe 39 (Martin), ED Protokoll Nr. 545.

${ }^{19} \mathrm{Im}$ Wintersemester 1892/93 waren es lediglich 8 Studenten gewesen. Zwischen 1902 und 1905 stieg die Zahl auf durchschnittlich 70 Hörer. Die Zahl der Kursbesucher verdreifachte sich im gleichen Zeitraum.

(Zur Teilnehmerfrequenz vgl. Staatsarchiv des Kantons Zürich: U 110 .)

\section{Summary}

The history of european physical anthropology in the nineteenth century was partly marked by the emergence of new institutions and the beginning of professionalisation. The aim to establish anthropology as a branch of university-education was not only put forward by anthropologists but the interest in ethnology and the racial research for colonial policy and foreign trade was an additional factor of considerable importance in view of research management and organization. In Zürich, the establishing of physical anthropology started by sporadic lectures at the University and at the ETH, followed by the foundation of the Ethnographische Gesellschaft in 1884. This led to an extraordinary professorship at the Philosophical Department of the University of Zürich and to the nomination of Rudolf Martin in 1899.

Dr. phil Hans-Konrad Schmutz

Naturwissenschaftliche Sammlungen der Stadt

Museumstraße 52

8400 Winterthur 